\title{
Gold nanoparticle-Quercetin composite formulation: A Computational Model Structure
}

\author{
Soumalya Chatterjee a, Debraj Hazra a, Rajat Pal a, ${ }^{*}$
}

\author{
a Department of Microbiology and Biotechnology, Sister Nivedita University, Kolkata-700156, West Bengal, India \\ *Corresponding author Email: rajat.p@snuniv.ac.in \\ DOI: https://doi.org/10.34256/nnxt2131
}

Received: 25-06-2021; Revised: 08-07-2021; Accepted: 08-07-2021; Published: 09-07-2021

Abstract: Nanoparticle mediated drug delivery is an emerging area of research now a days. In our present study, we emphasized on the mode of interaction of a widely used drug, Quercetin with frequently worked metallic nanoparticle, Gold (Au). At first five $-\mathrm{OH}$ groups have been attached separately with gold atom and energy minimization was performed using Avogadro Software for windows system. From this, we found that the $-\mathrm{OH}$ groups present at 7 position of ' $A$ ' ring, 3 ' and 4 ' positions of ' $B$ ' ring are most suitable site for gold atom to bind. In the next level of study, a gold atom has been interacted with two quercetin molecules at a time. The gold atom was attached to $-\mathrm{OH}$ group of 7 position of one quercetin molecule and $4^{\prime}$ position of the other. The calculated energy was found to be $482.319 \mathrm{KJ} / \mathrm{Mol}$. Further, gold atoms were interacted with all $-\mathrm{OH}$ groups of quercetin molecule at a time to see its stability and the structure was found to have quite stable with an energy level of $218.074 \mathrm{KJ} / \mathrm{Mol}$. Lastly we tried to make a quercetin-gold nanoparticle model structure which mimics the actual nanocomposite synthesized in vitro where one gold atom was interacted with two quercetin molecules and the other $-\mathrm{OH}$ groups of quercetin molecules were again attached with gold atoms. This structure possesses energy of $439.880 \mathrm{KJ} / \mathrm{Mol}$. The bond lengths and bond angle of interacting $\mathrm{C}, \mathrm{O}$ and $\mathrm{Au}$ atoms were measured to characterize the complex.

Keywords: Quercetin, Avogadro software, Nanoparticles, Nanocomposite structures

\section{Introduction}

Nanoparticles are the most talked about subject for research in various fields now a day. These are very small particle having size ranges between 1 to $100 \mathrm{~nm}$ by at least one dimension. Humans can't see it with bare eyes. All Nanoparticles show various physical and chemical properties which differs from their larger material counterparts. Mostly the nanoparticles contain few hundreds of atoms. The material's properties change as the size of nanoparticles approaches to the atomic scale, this only happens due to the increase in surface area to volume ratio when compared to bulk materials, like powder, plate and sheet, paper etc. For this reason, the surface atoms of the materials dominate the material performance. Because of this feature, all nanoparticles show unexpected optical, physical and chemical properties as they are very small enough to confine the electrons and produce Quantum effects [1]. The vast applications of nanoparticles in various fields have drawn the focus on its research. In the sector of biomedicine and agriculture, nanoparticles perform the most important role. Some metals are found to be very good ingredient for synthesis of nanoparticles. Many metals have already been reported for being used as precursor for nanoparticle synthesis, among them some mostly common are Gold ( $\mathrm{Au}$ ) [2], Silver (Ag) [3], Copper (Cu) [4], Iron [5], Zinc (Zn) [6], Nickel (Ni) [7], Platinum (Pt) [8], Palladium (Pd) [9], Ruthenium (Ru) [10], Rhodium (Rh) [11], cadmium (Cd) [12] and antimony (Sb) [13]. The nanoparticles which are made from these metals are widely used as a vehicle for drug molecules to be administered during the treatment against several diseases. All that kind of supports of such vehicle makes this drug -nanoparticle composites very useful mode of drug delivery system in medical and agricultural science. Free drug molecules generally dissociate and metabolize very easily in animal body due to the presence of different metabolic pathways. But if these drug molecules are administered with a vehicle like nanoparticle, the slow release will occur in physiological condition and the drug will get more time to show its activity in the body. Hence this process becomes very useful for scientists who are working on drug -nanoparticles composite formulation and their application in various fields. 
Metal nanoparticles possess many, physical, chemical, electronic, electrical, mechanical, thermal, optical and biological characteristic features [14, 15]. For having these properties, nanoparticles are being used in a verity of areas in biology and medicine such as fluorescent labeling $[16,17,18]$, drug and gene delivery $[19,20]$, detection of pathogens [21] and proteins [22], probing of DNA structure [23], tissue engineering [24, 25], MRI contrast enhancement [26], phagokinetic studies [27] and many more. Nanoparticles must interact with drug molecules or any other ligand for their application.

For the treatment against various diseases, flavonoids stand in a strong position among different biomolecules having medicinal activity. So, the composite structure of metal nanoparticles with different flavonoid molecules plays key role in the field of biomedical research [28]. According to many reports, the metal nanoparticles are synthesized by using the extracts of different parts of several plants. This method is commonly known as green synthesis [29].

Nanoparticles have been used as a vehicle system in Drug Delivery research for a long time. This vehicle system is composed of a composite structure of drug and nanoparticles which are attached together. To optimize the most suitable drug molecule with its perfect carrier nanoparticle we need to observe the interaction between them. For our study, we selected Quercetin as a drug molecule and gold nanoparticles as a career. Quercetin is a flavonoid molecule which has major array of applications in various fields and can act as, anti-inflammatory [30], antioxidant [31] and anticancer [32] agent. We selected this bioflavonoid because it contains five $-\mathrm{OH}$ groups which are well- distributed throughout its surface. From this study we can have a better and clear idea about the formation and development of metal nanocomposite structures.

\section{Methods}

In this present study, we selected Quercetin to be interacted with gold atoms for the formation of quercetin - gold nanocomposite structure. The molecular structure of quercetin was designed using Avogadro software for windows [33] and subjected for energy minimization. After energy minimization, the model structure along with the chemical structure of Quercetin has been shown in figure 1. In the structure of this drug molecule, $5-\mathrm{OH}$ groups are present that means there are 5 probable binding sites for metal nanoparticles for the formation of nanocomposite structures. We selected a single gold atom for the formation of nanocomposite structure. A nanoparticle is consisting of many gold atoms which is not possible to generate by computational method, hence we interacted a single gold atom.

Here, we performed our experiments in four segments. At first, we performed the interaction of gold atoms with all $-\mathrm{OH}$ groups present in the drug molecule separately one by one. Hence, we generated 5 nanocomposite model structures. After that we interacted five gold atoms with all $5-\mathrm{OH}$ groups of quercetin drug molecule at a time and all five gold atoms were distributed throughout the surface of drug molecule as all $-\mathrm{OH}$ groups are spread over the molecule. Not only that, we also tried to attach two drug molecules with a single gold atom. In the later structure, one gold atom holds two individual molecules of the drug.

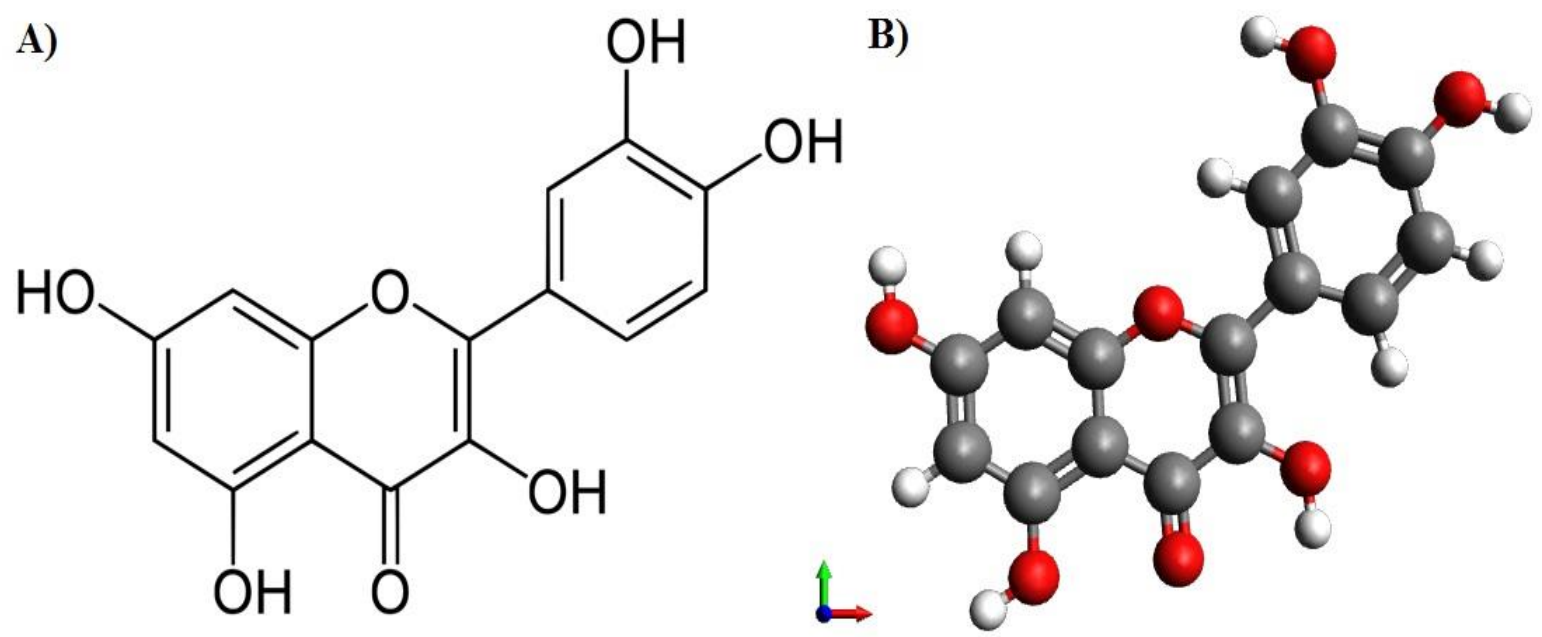

Figure 1. A) Chemical structure of quercetin and B) Model structure of quercetin constructed by Avogadro software. 
After that we combine it to the third level of study, i.e., not only one gold atom has been interacted with two drug molecules but also the other free $-\mathrm{OH}$ groups of the molecule were interacted with gold atoms. By doing this model, we tried to mimic the actual drug-nanocomposite model structure. In experimental condition, drug-nanocomposite structures may have multiple drug molecules to be interacted with multiple metal atoms. That means, one metal atom can be interacted with two drug molecules and each drug molecules may be interacted with many metal atoms. Hence from this study, we formulated the drugnanocomposite model structure which may represent the experimentally synthesized drug-nanocomposite structures. After the formation of all drugnanocomposite model structures, they were subjected for energy minimization by the same software. After that, the model structure with lowest energy level was considered for the most stable structure.

\section{Results}

As mentioned in the method section, first we attached one gold atom to each $-\mathrm{OH}$ group of quercetin molecule separately. As five $-\mathrm{OH}$ groups are present in the structure of Quercetin, five nanocomposite structures were formulated. All these structures are depicted in figure 2. As discussed in method for first level of study, the gold atom will be attached at 7, 5, 3, 4' and $3^{\prime}$ positions of quercetin in five different nanocomposite structures. These structures are named as Au_Q_1, Au_Q_2, Au_Q_3, Au_Q44 and Au_Q_5. The energy levels of all these structures are listed in table 1.
A)
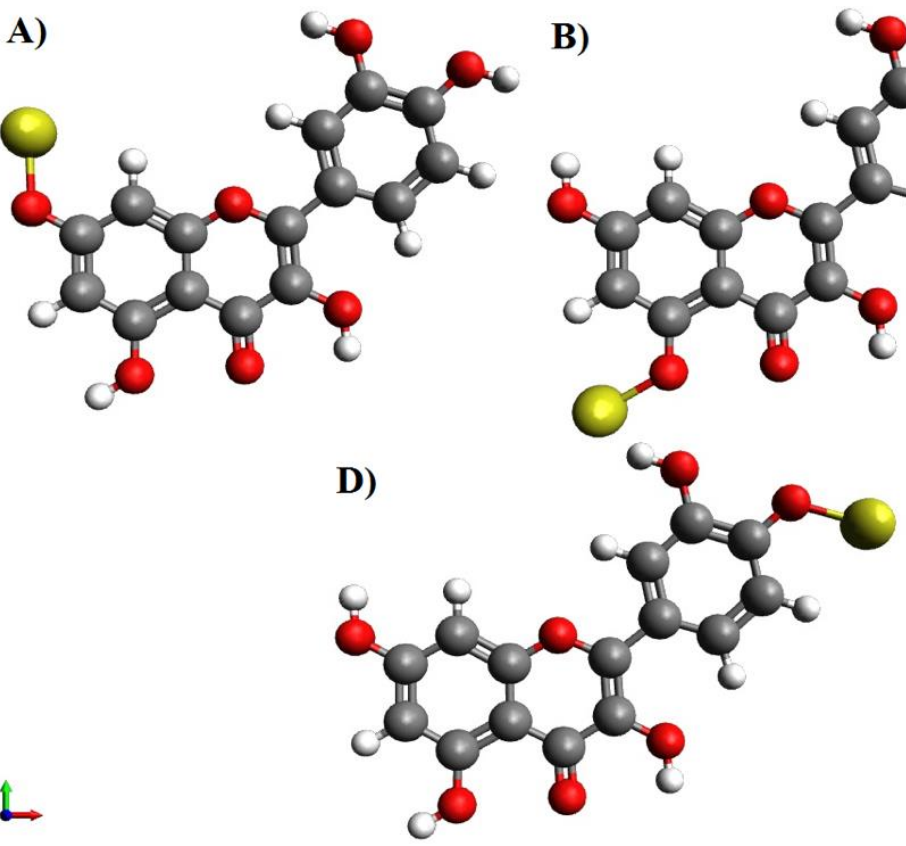

B)

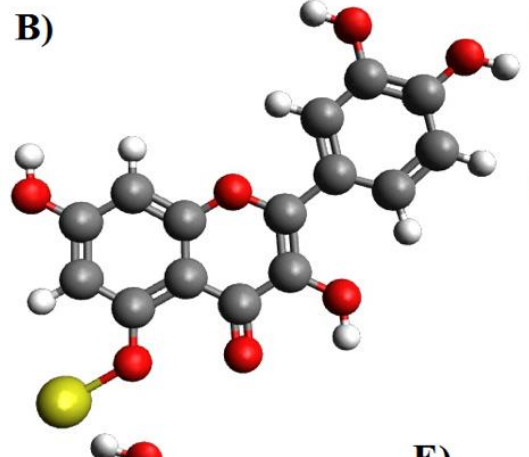

E)

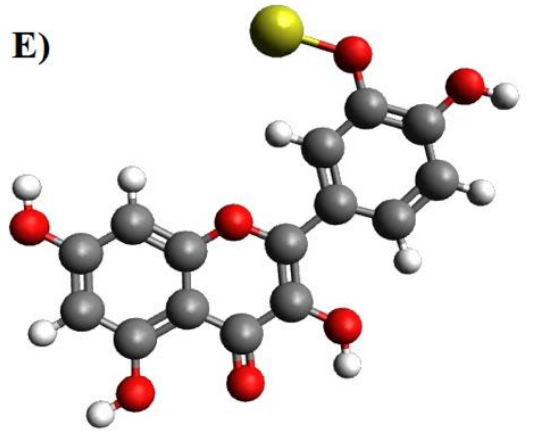

C)

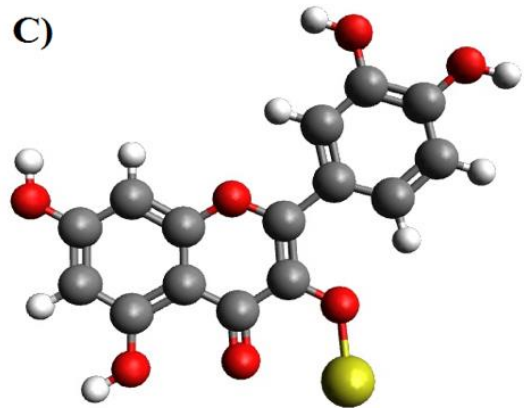

Figure 2. Nanocomposite model structure of A) Au_Q_1, B) Au_Q_2, C) Au_Q_3, D) Au_Q_4 and E) Au_Q_5.

Table 1. The list of energy levels of different nanocomposite structures of Quercetin with Gold atom when attached at different $-\mathrm{OH}$ groups separately.

\begin{tabular}{|l|l|l|}
\hline $\begin{array}{c}\text { DRUG } \\
\text { MOLECULE }\end{array}$ & Compound & Energy (KJ/Mol) \\
\hline Quercetin & Au_Q_1 & 226.224 \\
& Au_Q_2 & 226.251 \\
\hline & Au_Q_3 & 228.138 \\
\hline & Au_Q_4 & 226.231 \\
\hline & Au_Q_5 & 226.225 \\
\hline
\end{tabular}



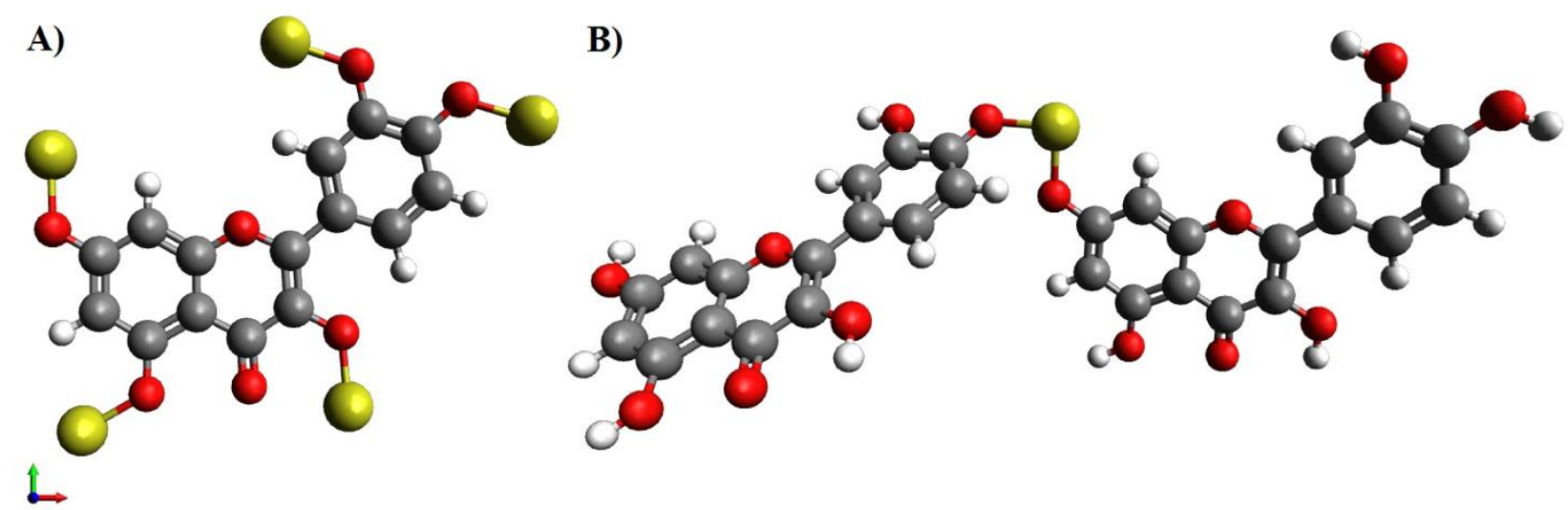

Figure 3. Nanocomposite model structure of A) Au_Q_6 and B) Au_Q_7.

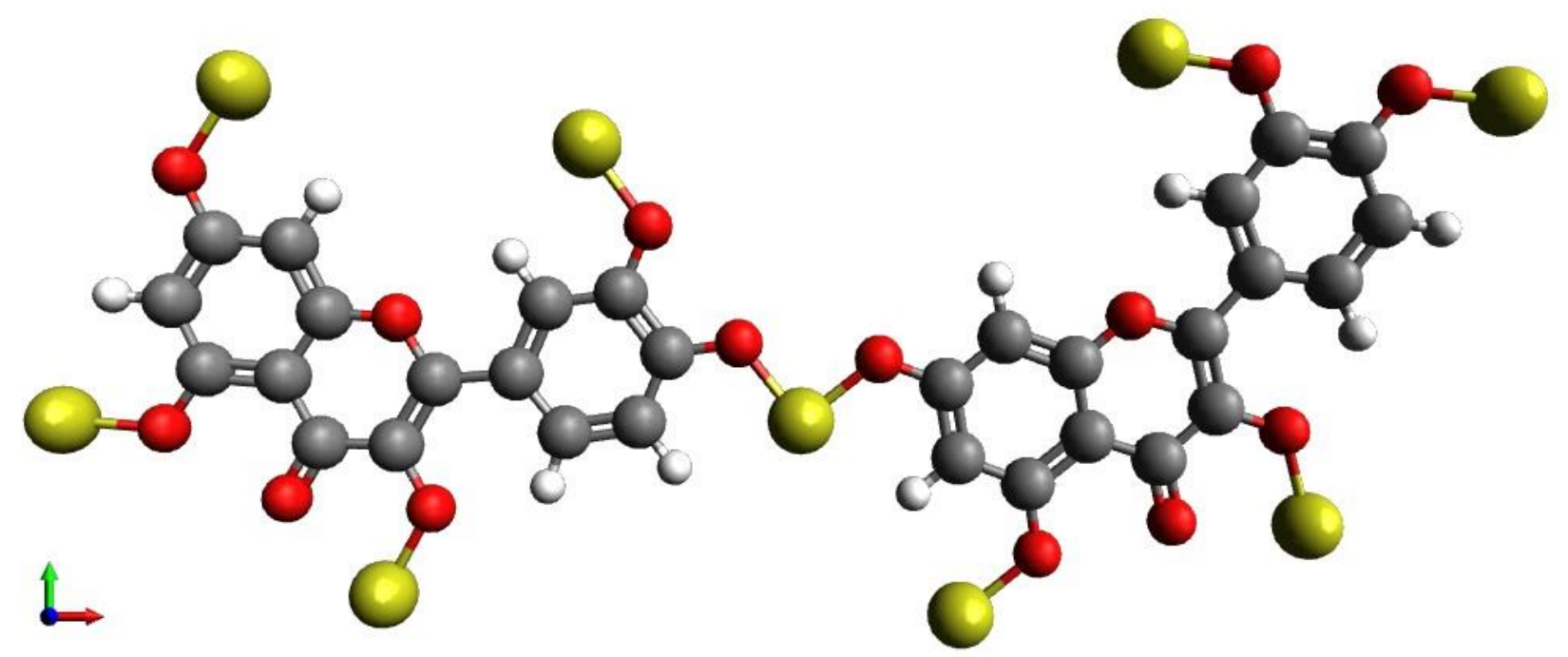

Figure 4. Nanocomposite model structure of Au_Q_8.

By comparing the energy levels, we can observe that the nanocomposite structure where the $\mathrm{Au}$ atom was attached at $-\mathrm{OH}$ group present at $4^{\prime}$ position of Quercetin was having lowest energy level suggesting most stable nanocomposite structure among all of these.

As quercetin has five - $\mathrm{OH}$ groups, in our next step, we attached five Au atoms with all -OH groups present in the drug molecule at a time and the composite structure is named as Au_Q_6 (figure 3A). After five gold atoms were interacted with all five $-\mathrm{OH}$ groups present in quercetin, the composite structure was subjected for energy minimization and the energy possessed by it was $218.074 \mathrm{KJ} / \mathrm{Mol}$. Surprisingly it is less than the previous structures suggesting more stability.

In a drug-nanocomposite formulation, as one drug molecule may interact with more than one nanoparticle, reverse is also true. That means one nanoparticle may also interact with more than one drug molecules. To make a representative model structure of this phenomenon, we constructed a nanocomposite where one $\mathrm{Au}$ atom is attached with two quercetin molecules. This nanocomposite model structure was named as Au_Q 7 and is depicted in figure $3 \mathrm{~B}$. In this structure one gold atom is tagged with two quercetin molecules through the most suitable $-\mathrm{OH}$ groups. After energy minimization, the structure exhibited energy level of $482.319 \mathrm{KJ} / \mathrm{Mol}$.

At the last level of study, we tried to formulate the actual model structure of gold-drug nanocomposite structures which may actually be formed during its chemical synthesis in vitro. At first one gold atom has been attached with two drug molecules and then many gold atoms were attached to the other free $-\mathrm{OH}$ groups of the drug molecules.

Quercetin has five free $-\mathrm{OH}$ groups amongst which one energetically favorable $-\mathrm{OH}$ for binding with gold, is the $-\mathrm{OH}$ present at $4^{\prime}$ position. Hence amongst all $-\mathrm{OH}$ groups, $4^{\prime}$ positioned $-\mathrm{OH}$ groups of two different drug molecules were interacted with one gold atom and other free $-\mathrm{OH}$ groups on the drug molecules in the composite were also attached with gold atoms forming a complex structure named Au_Q8 (Figure 4). This nanocomposite has energy level of $433.386 \mathrm{KJ} / \mathrm{Mol}$. As we mentioned before in our 
study, gold nanoparticles are being used as a vehicle for several drug molecules to apply in medicine to retain the drug molecule in the physiological condition. All these studies were done in vitro to synthesize, characterize and to estimate their application efficacy. But the study of interaction between the nanoparticle and drug molecule is not addressed well. Hence our study may put some light on this field of research and researchers in this area may have some idea about the selection of proper vehicle for a specific drug molecule. In our laboratory we have studied similar experiment previously to formulate these kinds of nanocomposite model structures [34]. Other groups [35, 36] have done research on the nanocomposite of quercetin and gold nanoparticle but they have only concentrated on the wet laboratory experiments not in the computational model for any interaction study. Hence out study will give a new direction for the future research on this.

\section{Conclusion}

Quercetin is a drug which contains five $-\mathrm{OH}$ groups hence can form five different nanocomposite structures with any metal when attached to different $\mathrm{OH}$ groups separately. Among all these $-\mathrm{OH}$ groups, the $-\mathrm{OH}$ present at $4^{\prime}$ position of the drug molecule showed lowest energy when interacted with gold metal. This suggests that $-\mathrm{OH}$ at carbon number $4^{\prime}$ might be the most suitable position for nanoparticle attachment. The final structure was formed where one gold atom is attached with two quercetin molecule and other free $-\mathrm{OH}$ groups of quercetin again attached with gold atoms. From this study it can be concluded that Gold atoms can bind with the -OH groups of quercetin molecule. The most suitable sites for gold atom binding are $7,5,4^{\prime}$ and $3^{\prime}$ positioned $-\mathrm{OH}$ in quercetin molecule but not the $-\mathrm{OH}$ present at 3 position. When gold atoms bind, the energy level gets lower suggesting more stability of the nanocomposite. A representative quercetin - gold nanocomposite model structure has been designed in silico having energy level of $433.386 \mathrm{KJ} / \mathrm{Mol}$.

\section{References}

[1] Twi-global, (2021) Technical knowledge of Nanoparticle, https://www.twiglobal.com/technical-knowledge/faqs/whatare-nanoparticles.

[2] B. Duncan, C. Kim, V. M. Rotello, Gold nanoparticle platforms as drug and biomacromolecule delivery systems, Journal of

Controlled Release, 148 (2010) 122-127. [DOI]

[3] C. A. D. Santos, M. M. Seckler, A. P. Ingle, I. Gupta, S. Galdiero, M. Galdiero, A. Gade, M. Rai, Silver Nanoparticles: Therapeutical Uses, Toxicity, and Safety Issues, Journal of Pharmaceutical Sciences, 103 (2014) 19311944. [DOI]

[4] T. Kruk, K. Szczepanowicz, J. Stefanska, R. P. Socha, P. Warszynski, Synthesis and antimicrobial activity of monodisperse copper nanoparticles, Colloids and Surfaces B: Bio interfaces, 128 (2015) 17 - 22. [DOI]

[5] S. A. Mahdy, Q. J. Raheed, P. T. Kalaichelvan, Antimicrobial Activity of zero-valent Iron Nanoparticles, International Journal of Modern Engineering Research, 2 (2012) 578-581.

[6] S. Rojas, F. J. Carmona, C. R. Maldonado, P. Horcajada, T. Hidalgo, C. Serre, J. A. R. Navarro, E. Barea, Nanoscaled Zinc Pyrazolate Metal-Organic Frameworks as Drug-Delivery Systems, Inorganic Chemistry, 55 (2016) 2650-2663. [DOI]

[7] D. Guo, C. Wu, J. Li, A. Guo, Q. Li, H. Jiang, B. Chen, $X$. Wang, Synergistic Effect of Functionalized Nickel Nanoparticles and Quercetin on Inhibition of the SMMC-7721 Cells Proliferation, Nanoscale Research Letters, 4 (2009) 1395-1402. [DOI]

[8] J. Kim, T. Shirasawa, Y. Miyamoto, The effect of TAT conjugated platinum nanoparticles on lifespan in a nematode Caenorhabditis elegans model, Biomaterials, 31 (2010) 5849-5854. [DOI]

[9] C. P. Adams, K. A. Walker, S. O. Obare, K. M. Docherty, Size-Dependent Antimicrobial Effects of Novel Palladium Nanoparticles, PlosOne, 9 (2014) e85981. [DOI]

[10] L. Xu, D. Liu, D. Chen, H. Liu, J. Yang, Size and shape controlled synthesis of rhodium nanoparticles, Heliyon, 5 (2019) e01165. [DOI]

[11] G. Viau, R. Brayner, L. Poul, N. Chakroune, E. Lacaze, F. F. Vincent, F. Fievet, Ruthenium Nanoparticles: Size, Shape, and Self Assemblies, Chemistry of Materials, 15 (2003) 486 - 494. [DOI]

[12] L. Qi, H. Colfen, M. Antonietti, Synthesis and Characterization of CdS Nanoparticles Stabilized by Double-Hydrophilic Block Copolymers, Nano Letters, 1 (2001) 61-65. [DOI] 
[13] W. Yin, W. Chai, K. Wang, W. Ye, Y. Rui, B. Tang, Facile synthesis of $\mathrm{Sb}$ nanoparticles anchored on reduced graphene oxides as excellent anode materials for lithium-ion batteries, Journal of Alloys and Compounds, 797 (2019) 1249-1257. [DOI]

[14] G. Schmid, Large clusters and colloids. Metals in the embryonic state, Chemical Review, 92 (1992) 1709 - 1727. [DOI]

[15] M.C. Daniel, D. Astruc, Gold Nanoparticles: Assembly, Supramolecular Chemistry, Quantum-Size-Related Properties and Applications toward Biology, Catalysis and Nanotechnology, Chemical Review, 104 (2004) 293 - 346. [DOI]

[16] M. Bruchez, M. Moronne, P. Gin, S. Weiss, A.P. Alivisatos, Semiconductor nanocrystals as fluorescent biological labels, Science, 281 (1998) 2013 - 2016. [DOI]

[17] W.C.W. Chan, S.M. Nie, Quantum dot bioconjugates for ultrasensitive nonisotopic detection, Science, 281 (1998) 2016 -2018. [DOI]

[18] S. Wang, N. Mamedova, N.A. Kotov, W. Chen, J. Studer, Antigen/antibody immunocomplex from CdTe nanoparticle bioconjugates, Nano Letters, 2 (2002) 817 - 822. [DOI]

[19] C. Mah, I. Zolotukhin, T.J. Fraites, J. Dobson, C. Batich, B.J. Byrne, Microsphere-mediated delivery of recombinant AAV vectors in vitro and in vivo, Molecular Therapy, 1, (2000) S239.

[20] D. Panatarotto, C.D. Prtidos, J. Hoebeke, F. Brown, E. Kramer, J.P. Briand, S. Muller, M. Prato, A. Bianco, Immunization with peptidefunctionalized carbon nanotubes enhances virus-specific neutralizing antibody responses, Chemistry and Biology, 10 (2003) 961 - 966. [DOI]

[21] R.L. Edelstein, C.R. Tamanaha, P.E. Sheehan, M.M. Miller, D.R. Baselt, L.J. Whitman, R.J. Colton, The BARC biosensor applied to the detection of biological warfare agents, Biosensors and Bioelectronics, 14 (2000) 805 813. [DOI]

[22] J.M. Nam, C.C. Thaxton, C.A. Mirkin, Nanoparticles-based bio-bar codes for the ultrasensitive detection of proteins, Science, 301 (2003) 1884 - 1886. [DOI]

[23] R. Mahtab, J.P. Rogers, C.J. Murphy, Protein sized quantum dot luminescence can distinguish between "straight", "bent", and "kinked" oligonucleotides, Journal of the Americal Chemical Society, 117 (1995) 9099 9100. [DOI]

[24] J. Ma, H. Wong, L.B. Kong, K.W. Peng, Biomimetic processing of nanocrystallite bioactive apatite coating on titanium, Nanotechnology, 14 (2003) 619 - 623.

[25] A. Isla, W. Brostow, B. Bujard, M. Estevez, J.R. Rodriguez, S. Vargas, V.M. Castano, Nanohybrid scratch resistant coating for teeth and bone viscoelasticity manifested in tribology, Materials Research Innovations, 7 (2003) 110-114. [DOI]

[26] R. Weissleder, G. Elizondo, J. Wittenburg, C.A. Rabito, H.H. Bengele, L. Josephson, Ultrasmall superparamagnetic iron oxide: characterization of a new class of contrast agents for MR imaging, Radiology, 175 (1990) 489 - 493. [DOI]

[27] W.J. Parak, R. Boudreau, M.L. Gros, D. Gerion, D. Zanchet, C.M. Micheel, S.C. Williams, A.P. Alivisatos, C.A. Larabell, Cell motility and metastatic potential studies based on quantum dot imaging of phagokinetic tracks, Advanced Materials, 14 (2002) 882 - 885. [DOI]

[28] S. Riaz, N. F. Rana, I. Hussain, T. Tanweer, A. Nawaz, F. Menaa, H. A. Janjua, T. Alam, A. Batool, A. Naeem, M. Hameed, S. M. Ali, Effect of Flavonoid-Coated Gold Nanoparticles on Bacterial Colonization in Mice Organs, Nanomaterials, 10 (2020), 1769. [DOI]

[29] A. M. E. Shafey, Green synthesis of metal and metal oxide nanoparticles from plant leaf extracts and their applications: A review, Green Processing and Synthesis, 9 (2020) $304-339$. [DOI]

[30] M. Lesjak, I. Beara, N. Simin, D. Pintac, T. Majkic, K. Bekvalac, D. Orcic, N. Mimica Dukic, Antioxidant and anti-inflammatory activities of quercetin and its derivatives, Journal of Functional Foods, 40 (2018) $68-75$. [DOI]

[31] W. Y. Oh, P. Ambigaipalan, F. Shahidi, Preparation of quercetin esters and their antioxidant activity, Journal of Agricultural and Food Chemistry, 67 (2019) 10653-10659. [DOI]

[32] L. Gibellini, M. Pinti, M. Nasi, J.P. Montagna, S. De Biasi, E. Roat, L. Bertoncelli, E.L. Cooper, A. Cossarizza, Quercetin and cancer chemoprevention, Evidence-Based Complementary and Alternative Medicine, 2011 (2011) 591356. [DOI] 
[33] M. D. Hanwell, D. E. Curtis, D. C. Lonie, T. Vandermeersch, E. Zurek, G. R. Hutchison, Avogadro: an advanced semantic chemical editor, visualization, and analysis platform, Journal of Cheminformatics, 4 (2012) 17. [DOI]

[34] S. Chatterjee, D. Hazra, R. Pal, In search of metal for construction of metal - Ferulic acid nanocomposite structure by in silico method, International Journal of Current Research, 13(5) (2021) 17585 - 17589. [DOI]

[35] M. Yilmaz, A. A. Karanastasis, M.V. Chatziathanasiadou, M. Oguz, A. Kougioumtzi, N Clemente, T. F. Kellici, N.E. Zafeiropoulos, A. Avgeropoulos, T. Mavromoustakos, U. Dianzani, S. Karakurt, A.G. Tzakos, Inclusion of Quercetin in Gold Nanoparticles Decorated with Supramolecular Hosts Amplifies Its Tumor Targeting Properties, ACS Applied Bio Materials, (2019) 2715-2725. [DOI]

[36] S. Balakrishnan, F. A. Bhat, P.R. Singh, S. Mukherjee, P. Elumalai, S. Das, C. R. Patra, J. Arunakaran, Gold Nanoparticle-conjugated quercetin inhibits epithelial-mesenchymal transition, angiogenesis and invasiveness via EGFR/ VEGRF 2 - mediated pathway in breast cancer, Cell Proliferation, 49 (2016) 678 - 697. [DOI]

\section{Funding}

No funding was received for conducting this study.

\section{Does this article screened for similarity?}

Yes

\section{Conflict of interest}

The authors have no conflicts of interest to declare that they are relevant to the content of this article.

\section{About the License}

(C) The author(s) 2021. The text of this article is open access and licensed under a Creative Commons Attribution 4.0 International License. 